\title{
Experimental Research of Dual-Polarization Passive Radar Based on DTTB Signal
}

\author{
Yonghu Zeng1, Xiaofeng Ai1,2, Liandong Wang1, Xiaoyang Wang1, Guangyong Zheng1 \\ ${ }^{1}$ State Key Laboratory of Complex Electromagnetic Environmental Effects on Electronics and Information \\ System, Luoyang 471003, China \\ ${ }^{2}$ National University of Defense Technology, State Key Laboratory of Complex Electromagnetic Environmental \\ Effects on Electronics and Information System, Changsha 410073, China \\ Email: anxifu2001@163.com
}

Received 13 December 2015; accepted 26 February 2016; published 2 March 2016

\begin{abstract}
The passive radar is a hot research topic. A multi-channel wideband passive radar experimental system is designed and the digital television terrestrial broadcasting (DTTB) signal is chosen to carry out the target detection experiment of civil aviation aircraft. The polarization and spatial filtering methods are used to solve the strong direct path interference suppression problems brought by the receiving system location; combined with the characteristics of DTTB signal, the block length selection interval in the block batch processing method for range-Doppler images calculation is given; the clutter suppression performance is compared through the experimental data receiving from different bistatic polarization channels, the conclusion is different from the monostatic radar and it can guide the passive radar experiment.
\end{abstract}

\section{Keywords}

Passive Radar, Clutter Suppression, Block Batch Processing, Dual-Polarization

\section{Introduction}

Passive radar, using transmissions of opportunity, has the potential advantages of anti-stealth and anti-jamming. In the last twenty years, the passive radar, based on radio and television, communication, navigation, wireless local area network signal and so on, has been researched widely. Many passive radar experimental systems have been developed both at home and abroad, and the basic theory and key technology of passive coherent radar have been made a great progress [1]-[4].

The typical passive radar system includes the "silent sentry" radar system developed by Martin Lockheed, and the HA100 radar system developed by Thales, France. In recent years, the digital broadcasting system, such as DVB-T, is widely used in the world. It has the advantages of wide range and remote distance of signal coverage, easy to obtain and easy processing. So the passive radar based on digital broadcasting signals has attracted wide attention of scholars all over the world [3]. China released the standard of Framing structure, channel coding and modulation for Digital Television Terrestrial Broadcasting (DTTB) system, with the independent intellectual 
property rights in 2006 [5]. This signal covers a wide area and has a wide bandwidth, so the use of this signal as a radiation source can obtain better detection ability and higher range resolution. Therefore, the passive radar based on DTTB signal has become an important research field [6]-[8].

Many radiation signals can be used for passive radar and the frequency range is very wide as above mentioned. Especially, the signal characteristics and waveforms of each frequency point are quite different from each other, so the corresponding signal processing method should be different. On the other hand, a wideband passive radar receiving system can be adapted to different scenarios if the working frequency can be set freely and the signal processing software can be re-constructed. For this purpose, a multi-channel wideband passive radar receiving and processing system is designed, each channel can be controlled independently, and the corresponding frequency band signal is acquired by center frequency selection. The general passive radar signal processing software depends on the Graphics Processing Unit (GPU) and Central Processing Unit (CPU) combined architecture and is realized by modularization, so it has the ability of reconstruction. Finally, the DTTB signal is chosen to carry out the detection experiment of civil aviation aircraft based on this experimental system.

\section{Receiving and Processing System}

This experimental system is designed with 6 channels, one of which is used as a reference channel and the other channels are used to form a surveillance array. The frequency-modulated radio (FM) and television (TV) signals can be used for target detection. In order to simplify the installation of antenna, the uniform linear array is used, and the internal between each array element can be adjusted flexibly. Uniform linear array has a series of advantages, such as compact structure, accurate measurement, etc. But the grating lobe is its major drawback, and it cannot solve the ambiguity as the passive receiver. Therefore, the directional antenna is used, and the main lobe of the array should cover the entire field of view. Under this condition, the grating lobe can be ignored, and the specific parameters depend on the field size of view and signal frequency.

The schematic diagram of the receiving channel is shown in Figure 1. The center frequency of the first band pass filter is $2.34 \mathrm{GHz}$, and the intermediate frequency of the second band pass filter is $70 \mathrm{MHz}$. The working bandwidth is about $1 \mathrm{GHz}$, and the receiver center frequency can be set arbitrarily. The receiving gains of the reference and surveillance channels are controlled independently, and the instantaneous bandwidth is $10 \mathrm{MHz}$, which covers most of the available signal. The digitizer board works in the Digital Down Comversion (DDC) mode, and the final sampling frequency of output is $10 \mathrm{MHz}$.

In order to suppress the direct wave in target detection by pattern synthesis, the self-calibration of channels is needed to eliminate the inconsistency of amplitude and phase. For this system, because the direct wave is strong in the reference channel, the direct wave is divided into several signals with the same amplitude and phase and injected into each surveillance channel, and then the errors of amplitude and phase can be compensated according to the output signal. Therefore, the radio frequency switch should be used for switching between the receiving channel and the calibration channel. The receiving and processing system is shown in Figure 2.

The GPU and CPU are combined for passive radar signal processing, which implement the high speed parallel computing capability of the GPU card, and the real-time performance of the radar signal processing is realized [10]. Through the mechanism of CPU and GPU combined processing, all signal processing algorithms are designed by the standard C programming language. The system functions can be reconstructed only by software reconstruction.

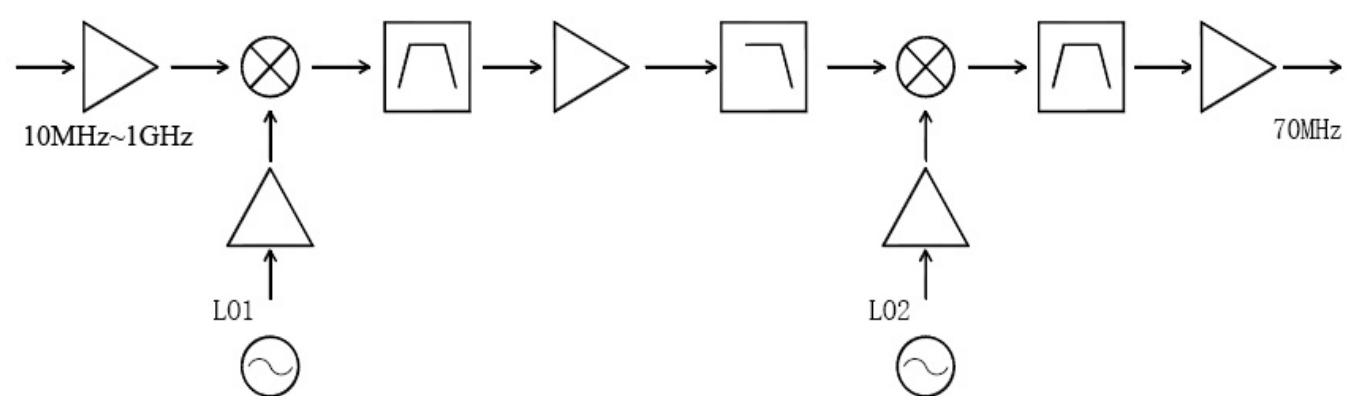

Figure 1. Block diagram of a receiving channel. 


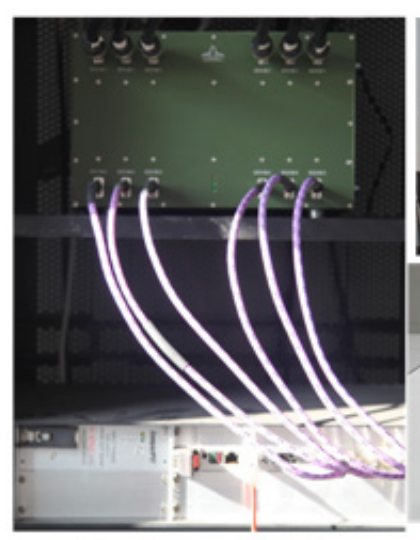

Receiver and DDC

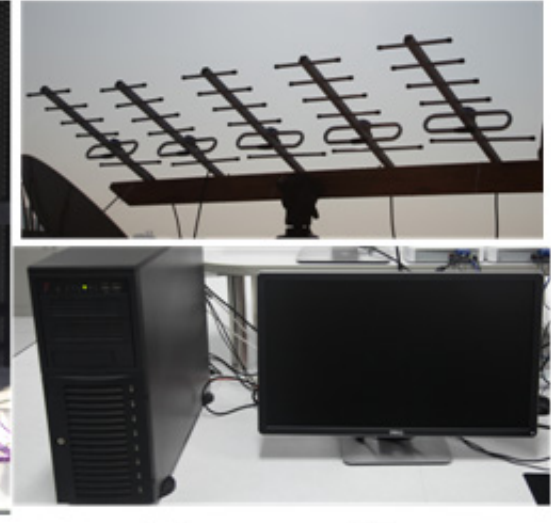

Antenna array and Server

Figure 2. The experimental system of DTTB-based passive radar.

In the application scenario, the signal of the transmitter should be intercepted firstly, and then the working frequency of the receiving system can be determined according to the parameters of the radiation source. Then, the antenna array geometry and beam forming algorithm should be determined according to the geometrical structure of the scene. The actual data processing can be carried out after above system parameters setting up. The basic data processing diagram is shown in Figure 3. After setting the frequency points, the reference signal purification and channel calibration should be carried out before target detection processing.

\section{Experimental Results and Analysis}

Compared with the FM radio, global system for mobile (GSM) and other signals, the transmitted power of DTTB signal is larger and the bandwidth is wider. Using the DTTB signal as the radiation source of the passive radar can achieve the longer detecting distance and higher range resolution, so it has been widely studied in several research institutions.

Firstly, the surrounding signal is analyzed by the spectrum analyzer and the corresponding DTTB signal frequency is found. The measured signal frequency spectrum is shown in Figure 4, and the center frequency is 682 $\mathrm{MHz}$ with $7.56 \mathrm{MHz}$ bandwidth. Then, the center frequency of the receiver is set accordingly. The detection performance is also compared with the track data provided by an Automatic Dependent Surveillance Broadcast (ADSB) transponder and recorded at the same time of the experiment.

\subsection{Direct Path Interference Suppression}

In the experiment, the distance of between the transmitter and the receiver is about $5 \mathrm{~km}$, so the direct path interference is so strong that the reference channel is easy to be in saturation state. As well known that the DTTB signal is transmitted with the horizontal polarization, and it is found that there is $10 \mathrm{~dB}$ difference between the received signals by horizontal and vertical polarized antenna in the reference channel, so the vertical polarized antenna is used for reducing the direct path interference.

In order to improve the weak signal receiving ability, the gain of the receiver should be set as the maximum, and the synthesis of an adapted pattern with a null in the direction of the reference illuminator. What's more, the fast block least mean square filter is used for further reduction of the direct path interference. This method has advantages of fast computation speed and fast convergence. The data of $0.4 \mathrm{~s}$ length is selected, and the adaptive filter iterative parameter is set as 0.006 and the filter length 512. The filter results of a test data is shown in Figure 6(a) and the cancelling gain achieves $24.4 \mathrm{~dB}$.

\subsection{Block Batch Processing}

The reference [9] proposed a block batch processing method for the passive radar signal processing according to the algorithm of the pulse Doppler radar. The algorithm needs to divide the reference and the surveillance signals into segments at the same time and it includes two steps of Fast Fourier Transform (FFT) and cross correlation, 


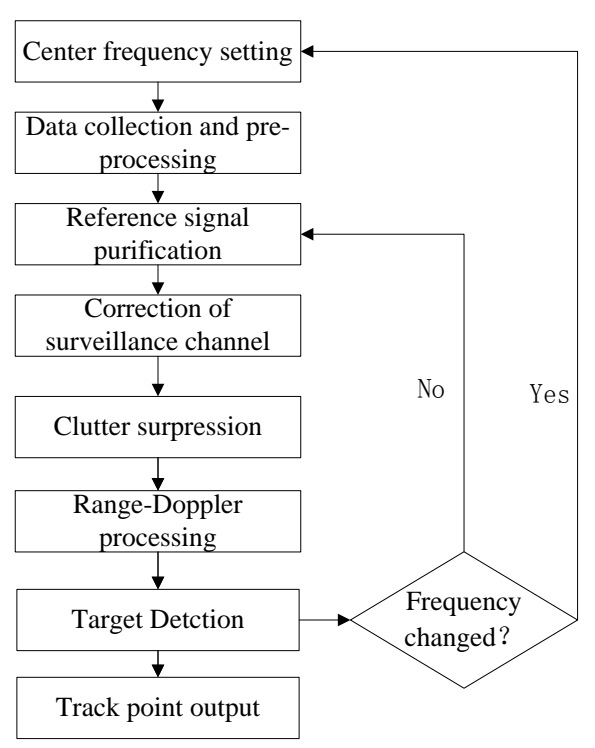

Figure 3. Block diagram of signal processing and system working.

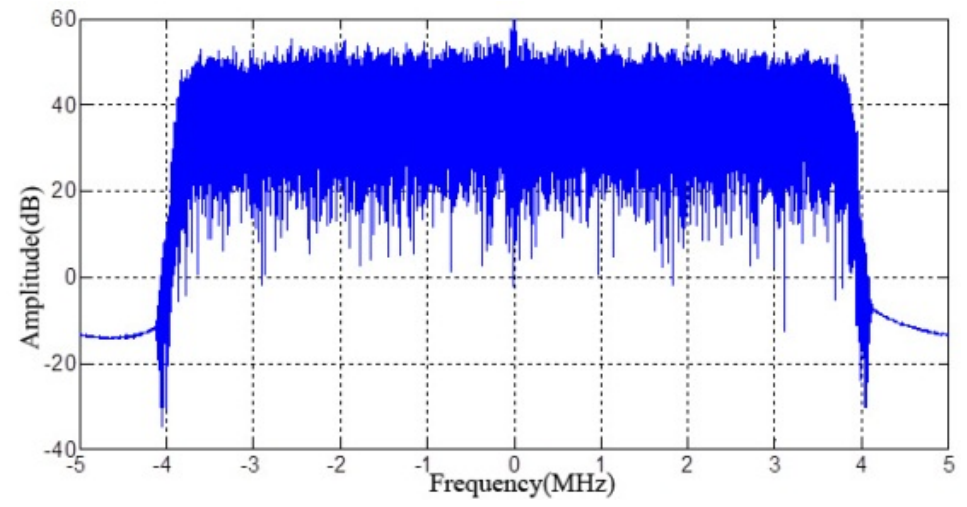

Figure 4. Frequency spectrum of the measured DTTB signal.

which is calculated by inverse FFT and FFT with high computation speed.

The block length is an important parameter in the algorithm. It is restricted by many factors, which can cause target lost or false target if the block length is not set appropriately. The block length determined by the maximum bistatic range, the maximum unambiguous bistatic velocity and no range migration. Through induce and analysis, the block length should meet the following conditions.

$$
\left\{\begin{array}{l}
l_{\text {seg }}<\frac{\lambda}{v_{\max }} f_{s} \\
L_{\text {seg }}=l_{\text {seg }}+\frac{R_{\max }}{c} f_{s}
\end{array}\right.
$$

where $f_{s}$ is the sampling frequency, $l_{\text {seg }}$ is block length of the reference signal, $L_{s e g}$ is block length of the surveillance signal, $\lambda$ is the wave length, $v_{\max }$ is the maximum bistatic velocity, $R_{\max }$ is the maximum bistatic range, $c$ is the light speed.

In addition, because the frame head is stronger than the frame content in the DTTB signal, it can be found that the longer the block length, the less the Doppler ambiguity of the signal itself. Figure $\mathbf{5}$ shows the range-Doppler images with different block lengths. When the block length is 6000 , only the real target can be seen in the range-Doppler image as shown in Figure 5(a), while the peak value caused by the signal itself ambiguity except the real target can be seen when the block length is 4500 . At this time, the false peaks should be removed as in 


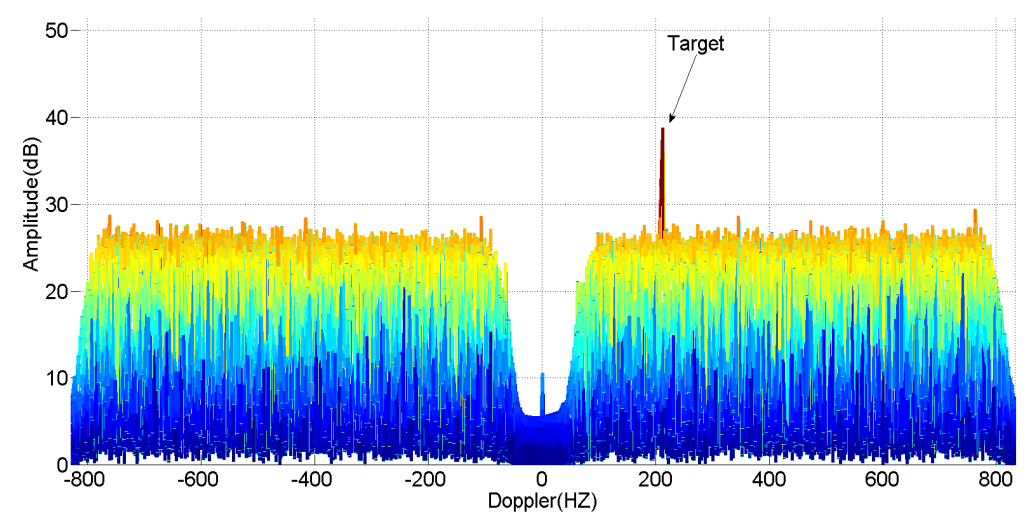

(a)

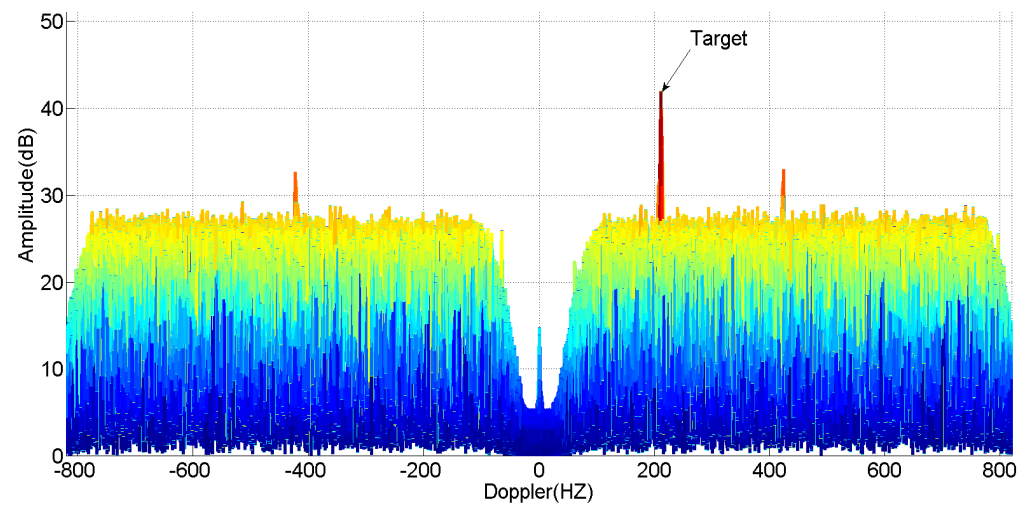

(b)

Figure 5. Range-Doppler images with different block lengths. (a) Block length of 6000; (b) Block length of 4500.

reference [6].

\subsection{Clutter Suppression Performance with Dual Polarization Channels}

Two surveillance channels are connected with horizontal and polarized antennas respectively, which correspond to the bistatic horizontal-vertical (HV) and horizontal-horizontal (HH) polarization receiving channels, and they point to the same direction. And then, the reference signal is used for clutter suppression processing with both surveillance channels, and the cancelling gains achieve $24.4 \mathrm{~dB}$ and $24.2 \mathrm{~dB}$, respectively.

The residual signals of the surveillance channels after clutter suppression processing are shown in Figure 6(a) and Figure 6(b). The results show that the $\mathrm{HH}$ channel has a higher residual signal fluctuation, although the cancelling gains are similar. That is to say the environment is sensitive to the receiving antenna polarization, and the clutter in HH channel should be stronger than the HV channel.

The range-Doppler images of the two channels are shown in Figure 6(c) and Figure 6(d), where areal target can be detected in the HV channel, while only the false targets caused by signal itself ambiguity in the HH channel. That is to say the direct wave is not cancelling completely, and the real target can't be detected in the HH channel. On the other hand, it also shows that the bistatic radar cross section (RCS) with HH polarization is less than the bistatic RCS with HV polarization, which leads to the weaker echo signal, so the target can't be detected in the HH channel. The experimental results show that the bistatic HV polarization is better for the civil airplane target detection than the bistatic $\mathrm{HH}$ polarization, which is different from the monostatic case.

\section{Conclusion}

The target detection experiment of dual polarization passive radar is carried out by using the broadband multi-channel receiving and processing system and the results show the availability of the experiment system. The 


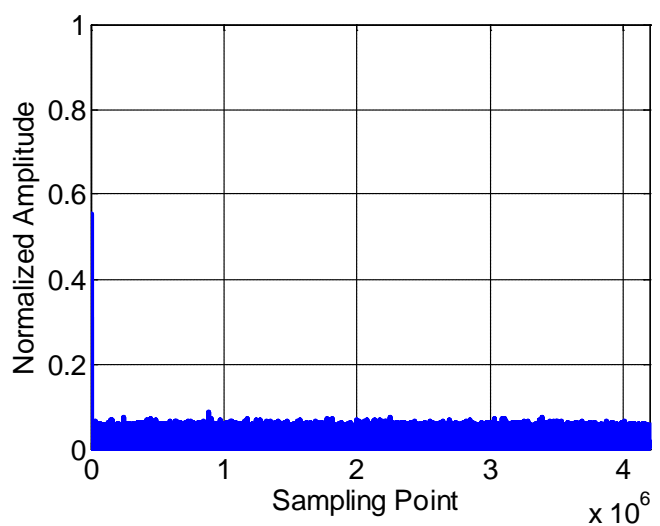

(a)

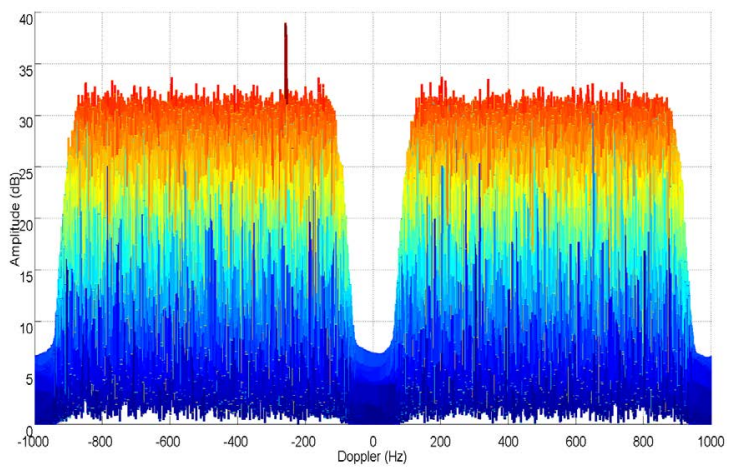

(a)

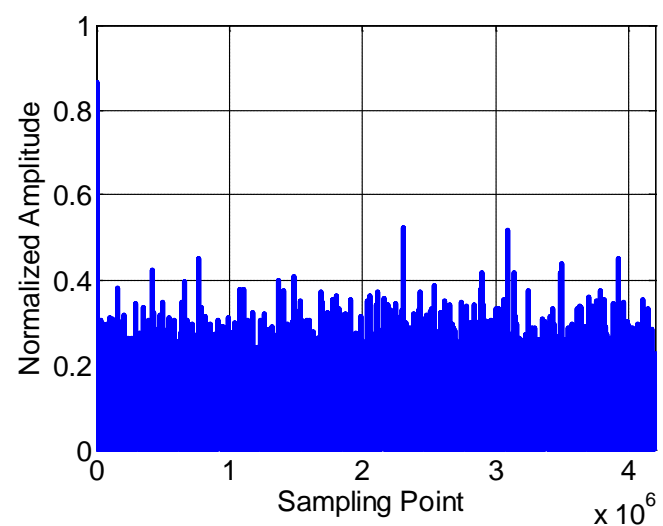

(b)

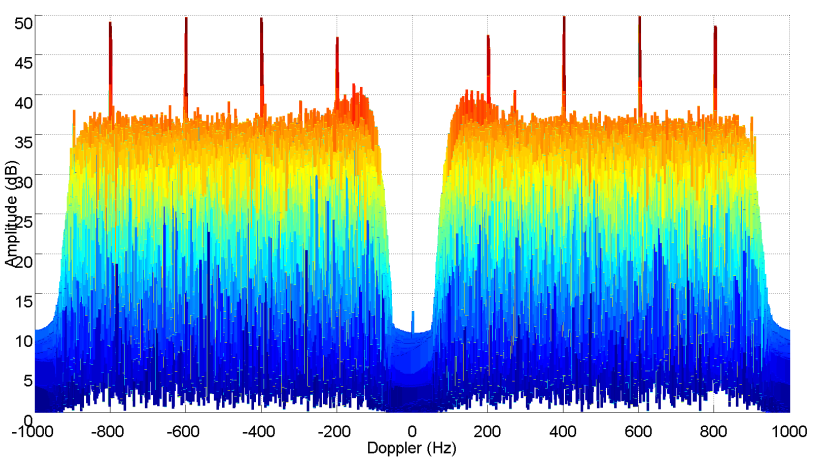

(b)

Figure 6. Clutter suppression results of different polarization channels.

strong direct wave suppression problem is solved by polarization and spatial filtering methods. The clutter suppression performances of different polarization channels are compared and the results show that the difference between the two channels is quite different. The further study should be on the measurement and statistical characteristics modeling of bistatic clutter and the target detection and identification of the dual-polarization passive radar.

\section{Acknowledgements}

This work is supported in part by the National Natural Science Foundation of China (No. 61401491).

\section{References}

[1] Song, J., He, Y., Cai, F.Q., et al. (2009) Overview of Passive Radar Technology Based on Non-Cooperative Radar Illuminator. Journal of Systems Engineering and Electronics, 31, 2151-2156.

[2] Wan, X.R. (2012) An Overview on Development of Passive Radar Based on the Low Frequency Band Digital Broadcasting and TV Signals. Journal of Radars, 1, 109-123. http://dx.doi.org/10.3724/SP.J.1300.2012.20027

[3] Shan, T., Liu, S.H., Zhang, Y.D., et al. (2015) Efficient Architecture and Hardware Implementation of Coherent Integration Processor for DVB-Based Passive Bistatic Radar. IET Radar Sonar \& Navigation, 9, 1-10.

[4] Tang, H., Wan, X.R., Chen, W.K., et al. (2013) Experimentation on Target Detection with Passive Radar Based on Digital Terrestrial Multimedia Broadcasting. Journal of Electronics \& Information Technology, 35, 575-580.

[5] Standardization Administration of the People's Republic of China (2006) GB (Chinese National Standard) 20600-2006: Framing Structure, Channel Coding and Modulation for Digital Television Terrestrial Broadcasting System.

[6] Gao, Z.W., Tao, R. and Wang, Y. (2009) Analysis and Side Peaks Identification of Chinese DTTB Signal Ambiguity Functions for Passive Radar. Science in China Series F: Information Sciences, 52, 1409-1417. http://dx.doi.org/10.1007/s11432-009-0149-y 
[7] Zhang, G.G., Wang, J. and Liu, Y.C. (2013) A Fast Cancellation Algorithm for DTTB-Based Passive Radar System. Journal of Electronics \& Information Technology, 35, 36-40. http://dx.doi.org/10.3724/SP.J.1146.2012.00669

[8] Jiang, B.F., Lv, X.D., Zhao, Y.D., et al. (2013) A Fast Algorithm of Ambiguity Function for Passive Coherent Radar Based on DTTB Signal. Journal of Electronics \& Information Technology, 35, 589-594. http://dx.doi.org/10.3724/SP.J.1146.2012.00900

[9] Chetty, K., Tan, B. and Woodbridge, K. (2014) Data Processing for Real-Time Wireless Passive Radar. IEEE Radar Conference, Cincinnati, OH, 19-23 May 2014, 455-459. http://dx.doi.org/10.1109/radar.2014.6875634

[10] Li, X.B., Guan, X., Zhong, L.H., et al. (2014) Real-Time Implementation of Signal Processing for Passive Radars Based on GPU. Journal of System Engineering and Electronics, 36, 2193-2198. 